\title{
Pulmonary Langerhans cell histiocytosis associated with Hodgkin's lymphoma
}

\section{To the Editor:}

Adult pulmonary Langerhans cell histiocytosis $(\mathrm{LCH})$ is a rare disorder of unknown aetiology that occurs predominantly in young smokers with a peak incidence occurring between 20 to 40 yrs of age. High-resolution computed tomography (HRCT) of the chest is essential for diagnosis and typically shows a combination of nodules and cavitated nodules in addition to thick- and thin-walled cysts. Few case reports of pulmonary LCH following diagnosis of Hodgkin's lymphoma (HL) have been described in the literature. In all but one case, the radiological description has mainly been based on standard chest radiographs with little attention paid to the smoking habits of these patients.

Here we describe two patients, one who developed pulmonary LCH simultaneously with HL and one who was diagnosed with pulmonary $\mathrm{LCH}$ during the follow-up examination for HL. Both of these patients show typical lung HRCT aspects. Interestingly, the two patients were heavy smokers at the time that the pulmonary $\mathrm{LCH}$ was diagnosed.

Although it is uncommon, physicians should keep in mind that pulmonary LCH should be considered as a differential diagnosis in the setting of diffuse cavitated nodules in patients with a history of HL.

The first case involved a 36-yr-old HIV-positive male who presented with symptoms including fever, weight loss, night sweats and enlarged peripheral lymph nodes in March 2003. Total body computed tomography (CT) revealed the presence of mediastinal and abdominal lymph nodes. A diagnosis of Hodgkin's lymphoma (HL) was established using a bone marrow biopsy, and the disease was classified as stage IV Bb. No pulmonary abnormalities were noted upon analysis of the thoracic CT scan at the time of the HL diagnosis. The patient received six cycles of MOPP/ABV until August 2003, resulting in the complete remission of HL, which showed no signs of involvement of the lung parenchyma upon analysis of the thoracic CT that was performed in October 2003. However, in February 2004 the patient complained of left thoracic pain and a moderate cough. A thoracic high-resolution CT (HRCT) subsequently revealed the presence of parenchymal nodules, some of which were cavitary, associated with the thin- and thick-walled cystic lesions and an osteolytic lesion on the sixth left rib (fig. 1a and b). Visual inspection of the bronchial tree was normal, and an extensive search for infectious agents was negative. Bronchoalveolar lavage recovered 430,000 cells $\cdot \mathrm{mL}^{-1}$ with the following differential cell counts: $90 \%$ macrophages, $9 \%$ lymphocytes and $1 \%$ neutrophils. Pulmonary function testing showed an increased residual volume $(149 \%$ of predicted), a forced expiratory volume in $1 \mathrm{~s}$ to vital capacity ratio equivalent to $76 \%$ and a diffusing capacity of the lung for carbon monoxide (DL,CO) of $82 \%$ pred. A total body CT evaluation and bone marrow biopsy did not show evidence of HL relapse. The diagnosis of Langerhans cell histiocytosis (LCH) was established by a biopsy of the rib lesion, which contained numerous CD1a+ cells associated with few lymphocytes, eosinophils and macrophages (fig. 1c and d). Interestingly, the patient's history revealed that he had smoked 15 cigarettes per day for a period of 20 yrs and that he had increased his smoking consumption to 25 cigarettes per day after he was diagnosed with HL. When the patient was informed that he had pulmonary and bone $\mathrm{LCH}$, he reduced his smoking consumption to five cigarettes per day. In November 2004, both the patient's lung HRCT and the residual volume (120\% pred) had improved significantly.

In our second case, a 29-yr-old female presented with symptoms including fatigue, weight loss, night sweats and enlarged cervical lymphadenopathies in June 2002. The diagnosis of nodular sclerosis HL was made upon performing a biopsy of a cervical lymph node, whereby the disease was classified as stage II Bb. At the time of the diagnosis, the analysis of the lung parenchyma on 5-mm thick sections using a thoracic CT was considered normal. The patient received seven courses of ABVD until February 2003, and complete remission of HL was obtained. In April 2004, while the patient was asymptomatic and her physical examination was normal, a routine follow-up chest radiograph revealed bilateral pulmonary abnormalities. A thoracic HRCT identified small pulmonary nodules, some of which were cavitary and associated with small cysts that predominated in the upper two-thirds of the lungs. The thoracic CT performed at the time of the HL diagnosis was then carefully reviewed, and a few scattered pulmonary nodules were retrospectively identified. These faint abnormalities had clearly increased in size between June 2002 and April 2004. A pulmonary function test was normal, except for the DL,CO (68\% pred). No extrapulmonary involvement of $\mathrm{LCH}$ was observed, and no additional infection was identified. Because the patient was asymptomatic and had no evidence of a relapse of $\mathrm{HL}$, a presumptive diagnosis of pulmonary $\mathrm{LCH}$ was made without histological confirmation. Interestingly, a precise history of the patient's smoking habits revealed that, while she used to smoke three cigarettes per day, after being informed that she had HL, she dramatically increased her cigarette consumption to two packs per day. In November 2004, while the patient had decreased her cigarette consumption down to five cigarettes per day, only the cysts persisted in the lung HRCT scan.

The coexistence of $\mathrm{LCH}$ and a malignant lymphoma, particularly HL, has been previously reported and characterised by different manifestations [1,2]. Most frequently, an accumulation 

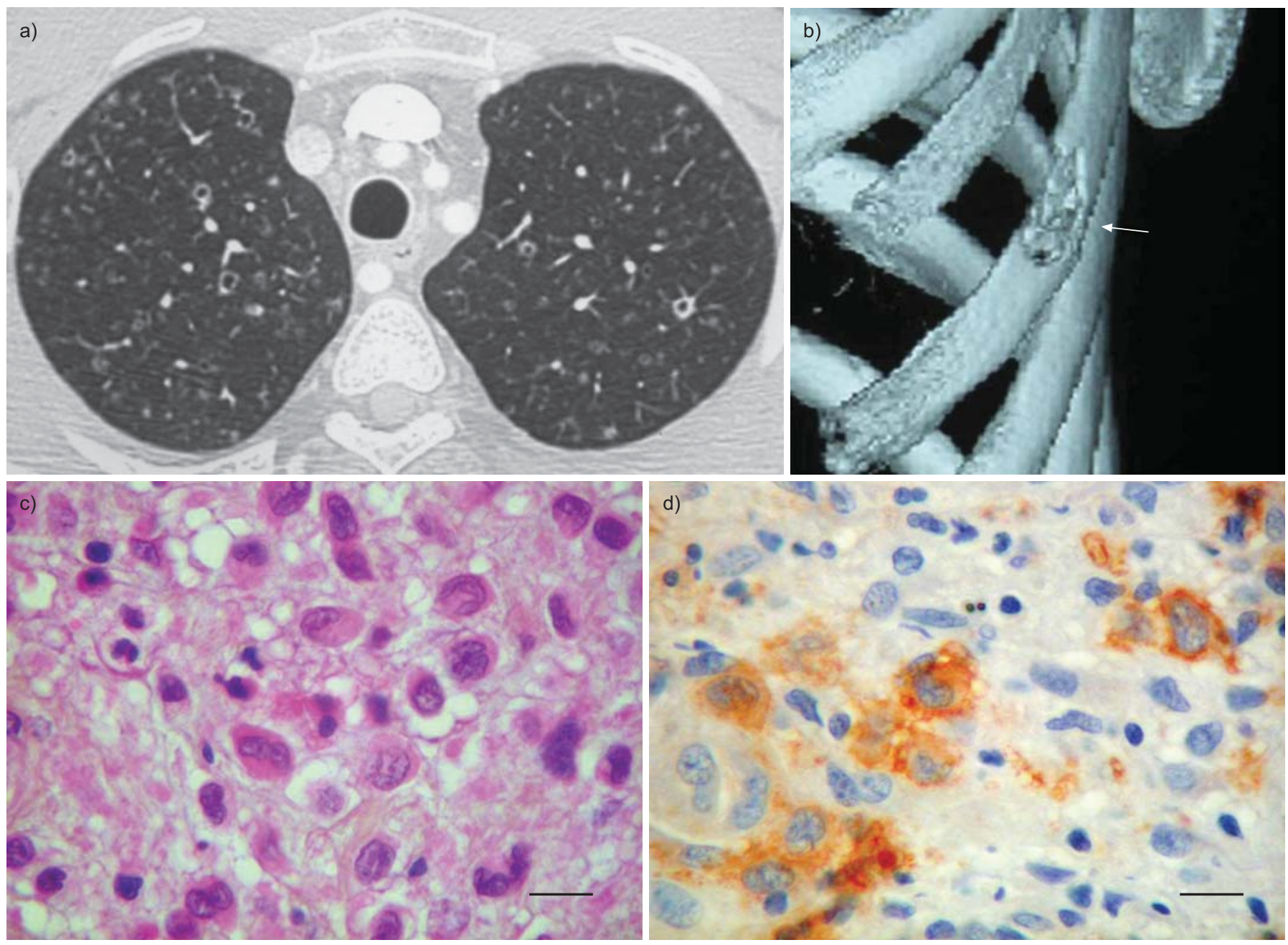

FIGURE 1. a) High-resolution computed tomography showing the typical association of nodules, cavitated nodules and thick- and thin-walled cysts of the lung from patient one, performed in February 2004 while he was in complete remission. b) Three-dimensional reconstruction of the patient's rib cage, showing an osteolytic lesion on the sixth left rib (arrow). Light microscopy image of the rib biopsy stained with haematoxylin and eosin, showing an infiltration by moderately sized cells with a convoluted irregular nucleus (c), which were positively stained using an antibody directed against CD1a (d). No Reed-Sternberg cells were identified. Scale bars=10 $\mu \mathrm{m}$.

of Langerhans cells is encountered at the site of the biopsy performed for the diagnosis of HL, or in other words, at the lymph node. This "LCH-like" localised reaction coupled with the pathological features of HL is usually considered a local immune response against tumour cells. In the vast majority of cases, no overt LCH disease is observed at a distant site unaffected by the lymphoma process. In a very small number of cases, however, the $\mathrm{LCH}$ disease has been reported in association with HL, involving the skin, bones or lungs [1, 3-8].

Surprisingly, although all series have clearly shown a strong correlation between pulmonary LCH and tobacco smoking, which probably plays a role in triggering the overt development of the disease [9], little attention has been paid to the smoking habits of patients with pulmonary LCH in the context of HL [1, $3-8]$. It is noteworthy that the two patients reported here were smoking heavily when the pulmonary LCH was discovered, as observed in patients with isolated pulmonary LCH.

A part from the role of tobacco smoking, host factors are clearly involved in the pathogenesis of pulmonary $\mathrm{LCH}$, as supported by the rarity of the disease relative to the number of smokers in the general population and the recurrence of the disease after lung transplantation [9]. Moreover, proliferative diseases, including haematological malignancies, are frequently reported in LCH patients [1], possibly because of shared genetic predisposition factors, as it has been recently suggested [10].

In summary, these two case reports emphasise the importance of lung HRCT in patients diagnosed with HL investigated for nodular pulmonary lesions. In patients with HL and appropriate genetic predisposition, exposure to tobacco probably plays a role in triggering the overt development of LCH in the lungs. Cessation of smoking and a careful follow-up examination without treatment seems suitable as an initial work-up in these patients.

\section{S. Feuillet*, L. Louis*, A. Bergeron*, A. Berezne", M-L} Dubreuil $^{\#}$, M. Polivka", E. Oksenhendler" and A. Tazi* *Université Paris 7, UFR Denis Diderot, Assistance PubliqueHôpitaux de Paris, Centre de Référence de l'Histiocytose 
Langerhansienne, Service de Pneumologie, Hôpital SaintLouis, "Université Paris 7, UFR Denis Diderot, Assistance Publique-Hôpitaux de Paris, Service d'Immunopathologie Clinique, Hôpital Saint-Louis, and "Université Paris 7, UFR Denis Diderot, Assistance Publique-Hôpitaux de Paris, Service d'Anatomie et Cytologie Pathologiques, Hôpital Lariboisière, Paris, France.

Correspondence: A. Tazi, Centre de Référence de l'Histiocytose Langerhansienne, Service de Pneumologie, Hôpital Saint-Louis, 1, avenue Claude Vellefaux, 75475, Paris cedex 10, France. E-mail: abdellatif.tazi@sls.aphp.fr

Statement of Interest: None declared.

Provenance: Submitted article, peer reviewed.

\section{REFERENCES}

1 Egeler RM, Neglia JP, Puccetti DM, et al. Association of Langerhans cell histiocytosis with malignant neoplasms. Cancer 1993; 71: 865-873.

2 Neumann MP, Frizzera G. The coexistence of Langerhans' cell granulomatosis and malignant lymphoma may take different forms: report of seven cases with a review of the literature. Hum Pathol 1986; 17: 1060-1065.

3 L'Hoste RJ Jr, Arrowsmith WR, Leonard GL, et al. Eosinophilic granuloma occurring in a patient with Hodgkin disease. Hum Pathol 1982; 13: 592-595.

4 Sajjad SM, Luna MA. Primary pulmonary histiocytosis $X$ in two patients with Hodgkin's disease. Thorax 1982; 37: 110-113.

5 Shanley DJ, Lerud KS, Luetkehans TJ. Development of pulmonary histiocytosis $\mathrm{X}$ after chemotherapy for Hodgkin disease. AJR Am J Roentgenol 1990; 155: 741-742.

6 Coli A, Bigotti G, Ferrone S. Histiocytosis X arising in Hodgkin's disease: immunophenotypic characterization with a panel of monoclonal antibodies. Virchows Arch A Pathol Anat Histopathol 1991; 418: 369-373.

7 Shin MS, Buchalter SE, Ho KJ. Langerhans' cell histiocytosis associated with Hodgkin's disease: a case report. J Natl Med Assoc 1994; 86: 65-69.

8 Unger JM, England DM, Collins J. Miliary nodules, Hodgkin's disease, and eosinophilic granuloma. J Thorac Imaging 1994; 9: 71-73.

9 Tazi A. Adult pulmonary Langerhans' cell histiocytosis. Eur Respir J 2006; 27: 1272-1285.

10 Rodig SJ, Payne EG, Degar BA, et al. Aggressive Langerhans cell histiocytosis following T-ALL: clonally related neoplasms with persistent expression of constitutively active NOTCH1. Am J Hematol 2008; 83: 116-121.

DOI: $10.1183 / 09059180.00007509$ 